\title{
Mental Health Problems of Individuals Under the Stay-Home Policy
}

\author{
Hayson Chenyu Wang ${ }^{1,2 *}$, Wenyun Ting ${ }^{1 *}$, Zhijin $\mathrm{Li}^{1}$, Elliot Tianyu Sun ${ }^{3}$, and Xiaojun Wang ${ }^{1 凶}$ \\ ${ }^{1}$ Peking Union Medical College Hospital, Chinese Academy of Medical Sciences and Peking Union Medical College, Beijing, China \\ 2John Radcliffe Hospital, University of Oxford, Oxford, UK \\ ${ }^{3}$ Radcliffe Department of Medicine, University of Oxford, Oxford, UK
}

With the pandemic of COVID-19, governments have successively adopted measures of asking people to reduce social activities, or staying at home entirely, which are indeed beneficial to the protection of susceptible people. ${ }^{1}$ However, at the same time, the mental health problems of the home-staying population have also increased. We conducted an online survey approved by the institutional ethics committee (S-K1173) investigating 2,392 home-staying Chinese individuals in the UK and China who were without a previous history of mental illness. All the participants were voluntarily involved. The results showed, during staying at home period, $60.11 \%$ experienced depression, $53.09 \%$ experienced sleep disturbance, $46.91 \%$ with irritability, and $48.2 \%$ with decreased libido. $76.12 \%$ of the individuals in the survey had sleep and circadian disorders. Nearly $80 \%$ of the people had indoor activities less than 30 minutes per day and $82.02 \%$ didn't do outdoor activities at all. $30 \%$ of people stayed single alone, lacking social support. The average time of the participants last left home was 5.47 days ago. From the promulgation of the stay-home policy to the present, the longest indoor stay time of the participants was 14.60 days on average, among whom, the maximum time of an individual staying indoors was 67 days. We also preliminarily noticed that the frequency and severity of these symptoms seem to be closely related to the time of staying indoors.

Many reasons may lead to mental health problems, among which it may be related to the panic of the disease or income reduction, but we excluded these individuals by the setting questions in the survey. Other reasons, like sleep and circadi-

Received: June 8, 2020 Revised: June 12, 2020

Accepted: June 18, 2020

$\bowtie$ Correspondence: Xiaojun Wang, $\mathrm{MD}, \mathrm{PhD}$

Peking Union Medical College Hospital, Chinese Academy of Medical Sciences and Peking Union Medical College, No.1 of Shuaifuyuan, District Dongcheng, Beijing 100730, China

Tel: +86-10-69158640, Fax: +86-10-69158640, E-mail: pumchwxj@yahoo.com

*These authors contributed equally to this work.

(a) This is an Open Access article distributed under the terms of the Creative Commons Attribution Non-Commercial License (https://creativecommons.org/licenses/bync/4.0) which permits unrestricted non-commercial use, distribution, and reproduction in any medium, provided the original work is properly cited. an disorders, lack of social support, and reduced physical activity, should be considered related to mental health problems, which we will try to discuss the specific mechanism by further studies.

Mental health problems affect individual health, such as the increased risk of metabolic diseases and reduced immunity, increasing the risk of COVID-19 infection. ${ }^{2}$ It may also affect family relations, as reported there has been a surge of divorce in China after locking down, ${ }^{3}$ leading to social problems. Mental health problems will have potentially negative feedback on the feelings of people on stay-home policy, decreasing their support for the epidemic prevention policies of governments, as well as the political and social stability. Under the current circumstances of lacking effective treatment and vaccines, stay-home policy or other policies that restrict social activities may be reserved longer in some countries. If so, governments or scientists should seek to countermeasure to minimize the impact of the mental health of individuals.

\section{Acknowledgments \\ None.}

\section{Conflicts of Interest}

The authors have no potential conflicts of interest to disclose.

\section{Author Contributions}

Conceptualization: Hayson Chenyu Wang, Xiaojun Wang. Data curation: Hayson Chenyu Wang, Wenyun Ting. Formal analysis: Zhijin Li. Investigation: Elliot Tianyu Sun. Software: Zhijin Li, Elliot Tianyu Sun. Supervision: Xiaojun Wang. Writing_-original draft: Hayson Chenyu Wang, Wenyun Ting. Writing_review \& editing: Xiaojun Wang.

\section{ORCID iDs}

Hayson Chenyu Wang

Wenyun Ting

Zhijin Li

Elliot Tianyu Sun

Xiaojun Wang

https://orcid.org/0000-0002-2958-1588 https://orcid.org/0000-0003-0689-5768 https://orcid.org/0000-0002-7060-8797 https://orcid.org/0000-0002-8245-0771 https://orcid.org/0000-0003-3043-0020

\section{REFERENCES}

1. Lippi G, Henry BM, Bovo C, Sanchis-Gomar F. Health risks and po- 
HC Wang et al.

tential remedies during prolonged lockdowns for coronavirus disease 2019 (COVID-19). Diagnosis (Berl) 2020;7:85-90.

2. Morey JN, Boggero IA, Scott AB, Segerstrom SC. Current directions in stress and human immune function. Curr Opin Psychol 2015;5:13-17.
3. Strain of life under lockdown sparks divorce surge in China. Financial Times. Available at: https://www.ft.com/content/11990ff0-c8f5-4f609b0a-be06324a4ddb. Accessed April 3, 2020. 